\title{
The kind of AKH-mobilized energy substrates in insects can be predicted without a knowledge of the hormone structure
}

\author{
Radomír SOCHA ${ }^{1}$, DALIBor KODRÍK ${ }^{1,2}$, Petr ŠIMEK ${ }^{1}$ and MARKÉTA PATOČKOVÁ ${ }^{1,2}$ \\ ${ }^{1}$ Institute of Entomology, Academy of Sciences, and ${ }^{2}$ Faculty of Biological Sciences, University of South Bohemia, Branišovská 31, \\ České Budějovice, 370 05, Czech Republic; e-mail: socha@entu.cas.cz
}

Key words. Adipokinetic hormones, Dysdercus cingulatus, Pyrrhocoris apterus, Locusta migratoria, Tenebrio molitor, Pyrap-AKH, lipids, carbohydrates, structure-function relations

\begin{abstract}
The aim of this study was to show that the kind of AKH-mobilized energy substrates in insects can be predicted on the basis of the results obtained with the application of heterologous, i.e. inter-species, AKHs. Four different AKHs, the Locmi-AKH-I inducing hyperlipaemia and hyperglycaemia in Locusta migratoria, Tenmo-HrTH inducing hyperglycaemia in Tenebrio molitor, and Pyrap-AKH and Peram-CAH-II inducing hyperlipaemia in Pyrrhocoris apterus were used, firstly in conspecific tests, secondly in all possible species-AKH combinations, and finally in individual applications on the test species, the cotton bug Dysdercus cingulatus. Since each of the AKHs induced hyperlipaemia in D. cingulatus adults, we predicted that lipids are the only energy substrates which are mobilized in this species by its native AKH. The accuracy of this prediction was subsequently confirmed by the structural identification of the native $D$. cingulatus AKH and conspecific application tests. The proposed methodical approach can serve as a suitable monitoring system for determination of the kind of energy substrates mobilized by native insect AKHs until the structure of the hormone is identified.
\end{abstract}

\section{INTRODUCTION}

The high energy demand during active flight (Goldsworthy, 1983) and walking (Socha et al., 1999; Kodrík et al., 2000) of insects is met by the oxidation of carbohydrates, lipids, the amino acid proline or mixtures thereof depending on the species (see reviews by Weeda et al., 1979; Beenakkers et al., 1984; Gäde \& Auerswald, 1998). The mobilization of stored fuels during episodes of flight or walking is controlled by peptides belonging to the socalled adipokinetic hormone family $(\mathrm{AKH})$. The neuropeptides of the family are usually octa-, nona- or decapeptides with both termini blocked (and one $11 \mathrm{mer}$ with nonamidated C-terminus - Köllisch et al., 2000), and are synthesized and released from the corpora cardiaca (CC). Besides the mobilization of energetic reserves these hormones exert additional physiological actions such as the inhibition of protein synthesis (Carlisle \& Loughton, 1979; Cusinato et al., 1991), the inhibition of mRNA synthesis (Kodrík \& Goldsworthy, 1995), the modulation of neuro- or myoactivity (Scarborough et al., 1984; Milde et al., 1995; Socha et al., 1999), and the inhibition of glycolysis (Becker \& Wegener, 1998) and lipid synthesis (Gokuldas et al., 1988; Ziegler, 1997; Lorenz, 2001). Moreover, the adipokinetic hormone of Locusta migratoria (L.), Locmi-AKH-I, has been shown to increase the immune response in locusts challenged with laminarin or bacterial lipopolysaccharide (Goldsworthy et al., 2002a, 2003). There are almost 40 isoforms (bioanalogues) of AKHs known from various representatives of all the main insect orders. For a review of the other common structural features see Gäde et al. (1997).

Different strategies have been used to analyze the metabolic functions of AKHs and/or isolate and characterize their molecular structures. A classical approach starts from the determination of the biological function of gland extracts and is followed by isolation and characterization of the AKH. Convenient heterologous bioassays, including tests of the crude extracts of CCs from tested insect species on the acceptor insects such as Locusta migratoria and/or Periplaneta americana, are commonly used for monitoring of their biological activities (Gäde et al., 1997). A more recent approach, taking profit of the chemical methods of analysis, firstly investigates the molecular structure of the hormone (usually by analogy) and then determines its function (Lafont, 2000). It is presumed that many peptide hormones have been conserved during evolution with function being less conserved than structure (Ebberink et al., 1989; Satake et al., 1999), a fact also apparent within vertebrate hormones. Various tissues may possess receptors for a given hormone and, therefore, this hormone will elicit different responses. However, it cannot be excluded that in the case of AKHs, the type of energy substrates mobilized can also be conservative due to evolutionarily pressures on a particular insect species. If this is correct, the AKHs originating from different insects can mobilize, in a test species on which they are applied, only the recipient-specific type of energy substrates used.

The aim of the present study was to test the hypothesis that the kind of AKH-mobilized energy substrates in an insect can be predicted on the basis of the results obtained with use of heterologous applications of AKHs. Provided that this approach is successful, then it can be used for quick screening and monitoring of the AKHs metabolic activity in insects whose native AKHs have not yet been identified. 


\section{MATERIAL AND METHODS}

\section{Experimental animals}

The firebug, Pyrrhocoris apterus, originating from wild populations collected at České Budějovice (Czech Republic, $49^{\circ} \mathrm{N}$ ) (Socha \& Šula, 1996), was used in the present study. All stages from egg to adult were kept in small glass jars with linden seeds and water ad libitum at a constant temperature of $26 \pm 1^{\circ} \mathrm{C}$ and under long-day conditions (18L : 6D). Ten day old adult females were used for experiments (see Socha \& Kodrík, 1999).

A colony of the migratory locust, Locusta migratoria, was kept under crowded conditions at a temperature of $30-32^{\circ} \mathrm{C}$ with a $12 \mathrm{~h}$ photoperiod. The insects were reared on lettuce or grass, supplemented with bran. After the final moult, males and females were kept in separate cages. Adults aged about 15 days after adult eclosion were used in the experiments.

The mealworms, Tenebrio molitor, were purchased from a local pet shop. The culture was kept at about $25^{\circ} \mathrm{C}$ with a photocycle of $14 \mathrm{~L}: 10 \mathrm{D}$. Both the larvae and the adults were fed on oat flakes and bran. Adults aged 5-10 days after eclosion were used in the experiments.

The stock culture of the cotton bug, Dysdercus cingulatus, was kept in small glass jars with linden seeds and water ad libitum at constant temperature of $28 \pm 1^{\circ} \mathrm{C}$ and $12 \mathrm{~h}$ photoperiod. Adult females aged 8 days after adult eclosion were used in the experiments.

\section{Lipid and carbohydrate mobilization assays}

The AKHs were dissolved in $20 \%$ methanol in Ringer saline and their biological activity ascertained by the mobilization assays described previously (Kodrík et al., 2000, 2002), either based on the sulpho-phosphovanillin test (lipids) (Zöllner \& Kirsch, 1962) and/or on the phenol test (carbohydrates) (Montgomery, 1957). For simplicity just two representative doses (10 and $40 \mathrm{pmol}$ ) were injected for each tested AKH.

\section{AKH identification}

Extraction of the AKH from D. cingulatus CNS. To dissect undamaged $\mathrm{CC}$ and to avoid possible losses of the peptidic material from the glands, the tissue samples (CNS) consisted of the brain with CC and corpus allatum (CA) attached. Subsequently, the organs were homogenised in $80 \%$ methanol by sonication. The supernatant was then evaporated to dryness and the residue used either for HPLC or for LC/MS analyses.

HPLC analysis. HPLC analysis was performed on a MerckHitachi D-6000 chromatography system using HPLC System
Manager D-7000 software, at a flow rate of $0.8 \mathrm{ml} / \mathrm{min}$ and UV monitoring at $215 \mathrm{~nm}$. The sample was fractionated on the Chromolith Performance RP-18e column (Merck) $100 \times 4.6 \mathrm{~mm}$ with gradient $25-100 \% \mathrm{~B}$ in $40 \mathrm{~min},(\mathrm{~A}=0.11 \%$ TFA in water, $\mathrm{B}=0.1 \% \mathrm{TFA}$ in $40 \%$ acetonitrile). All fractions were then taken for the ELISA competitive tests (retention time of the synthetic Pyrap-AKH is 12.27 in this system). The area where AKH peptides are usually eluted was taken for LC/MS structural analysis.

ELISA. Determination of AKH presence in D. cingulatus CNS extract fractionated on HPLC was performed using the competitive ELISA according to our protocol (Goldsworthy et al., 2002b). Briefly, rabbit antibodies raised against Cys ${ }^{1}-$ Pyrap-AKH coupled to keyhole limpet haemocyanin (Sigma Genosys, Cambridge, UK) was diluted $1: 10000$ and applied on a 96-well microtitre plate (high binding Costar, Corning Incorporated, Corning, NY, USA). Tested samples, together with the biotinylated probes (BLAM-Cys ${ }^{1}$-Pyrap-AKH), were than added to specific wells. After competition for the binding sites on the IgG bound to the plates, a streptavidin conjugated with horseradish peroxidase solution (Vector Laboratories) diluted $1: 500$ in PBS-Tween was added. Finally, OPD (orthophenylenediamine) reagent was used for the visualization. The absorbance values were determined in a microtitre plate reader at $492 \mathrm{~nm}$. One row of each plate contained a dilution series of Pyrap-AKH for the construction of a competition curve: this included one well with no material competing with the probe to determine the maximum absorbance (MAX) and one well with an excess of 10 pmol Pyrap-AKH to determine the non-specific absorbance (NSA). The percentage competition for each sample was calculated as: $100-100 *($ absorbance of the sample - NSA)/(MAXNSA).

LC/MS. The primary sequence of the AKH-peptide was deduced from the electrospray $\mathrm{MS}^{\mathrm{N}}$ spectra obtained by collision-induced dissociation (CID) of the detected $\mathrm{MH}^{+}$ion and its product fragment ions by the procedure described elsewhere (Kodrík et al., 2000).

\section{Synthetic adipokinetic peptides}

The adipokinetic hormone of L. migratoria, code-named Locmi-AKH-I, was purchased from Novabiochem (Germany). The other hormones, Pyrap-AKH and Peram-CAH-II from $P$. apterus, and Tenmo-HrTh from T. molitor, were customsynthesized by PolyPeptide Laboratories s.r.o. (Praha, Czech Republic).

TABLE 1. The effect of AKH-peptides on mobilization of lipids and carbohydrates in haemolymph after the injection of intrinsic AKH. Controls were injected with saline only. Statistically significant differences at the $5 \%$ level (experimental vs. control) are indicated by asterisks. Values in bold and italics = preferable energetic source; numbers in brackets $=$ number of replicates.

\begin{tabular}{|c|c|c|c|c|c|c|}
\hline \multirow{2}{*}{$\frac{\text { Insect species }}{P . \text { apterus }}$} & \multirow{2}{*}{$\frac{\text { Tested peptide }}{\text { Pyrap-AKH }}$} & \multirow{2}{*}{$\frac{\text { Peptide dose }}{10 \mathrm{pmol}}$} & \multicolumn{2}{|c|}{ Lipids (mg/ml) } & \multicolumn{2}{|c|}{ Carbohydrates (mg/ml) } \\
\hline & & & $10.75 \pm 3.08 *$ & (8) & $0.50 \pm 0.86$ & (5) \\
\hline & & 40 pmol & $4.08 \pm 3.32 *$ & (6) & $-0.07 \pm 0.25$ & (8) \\
\hline & Peram-CAH-II & $10 \mathrm{pmol}$ & $8.98 \pm 1.99 *$ & (5) & $-0.32 \pm 1.65$ & (10) \\
\hline & & 40 pmol & $10.71 \pm 2.50 *$ & (8) & $0.09 \pm 0.15$ & (9) \\
\hline & & Control & $-1.68 \pm 3.12$ & (5) & $-0.13 \pm 0.16$ & (8) \\
\hline \multirow[t]{3}{*}{ T. molitor } & Tenmo-HrTh & $10 \mathrm{pmol}$ & $0.24 \pm 0.81$ & (9) & $6.07 \pm 1.42 *$ & (10) \\
\hline & & 40 pmol & $0.06 \pm 0.97$ & (10) & $8.32 \pm 2.45 *$ & (18) \\
\hline & & Control & $-0.07 \pm 0.83$ & (9) & $0.53 \pm 1.42$ & (10) \\
\hline \multirow[t]{3}{*}{ L. migratoria } & Locmi-AKH-I & $10 \mathrm{pmol}$ & $10.61 \pm 3.46 *$ & (5) & $2.82 \pm 1.63 *$ & (10) \\
\hline & & 40 pmol & $14.81 \pm 3.41 *$ & (8) & $1.53 \pm 1.11^{*}$ & (9) \\
\hline & & Control & $0.49 \pm 0.38$ & (7) & $-1.23 \pm 1.12$ & (8) \\
\hline
\end{tabular}




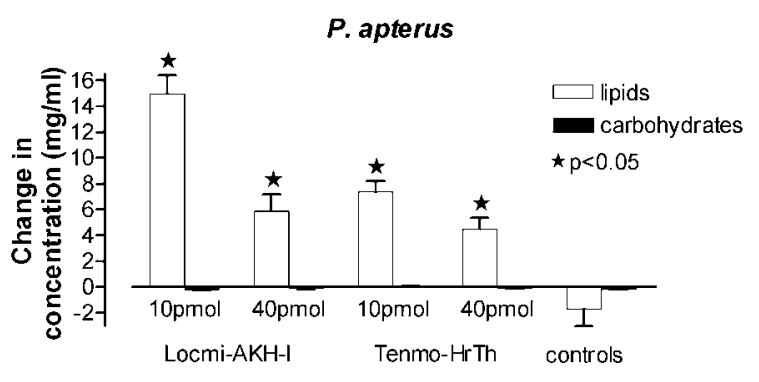

Dose of AKH peptide

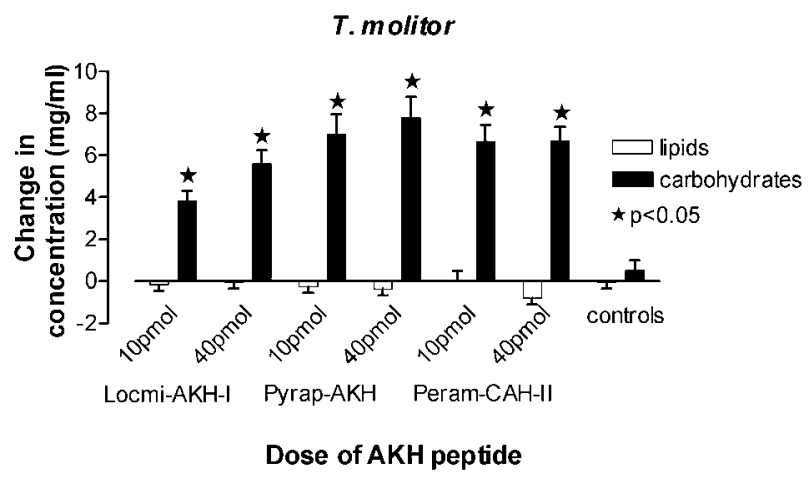

L. migratoria

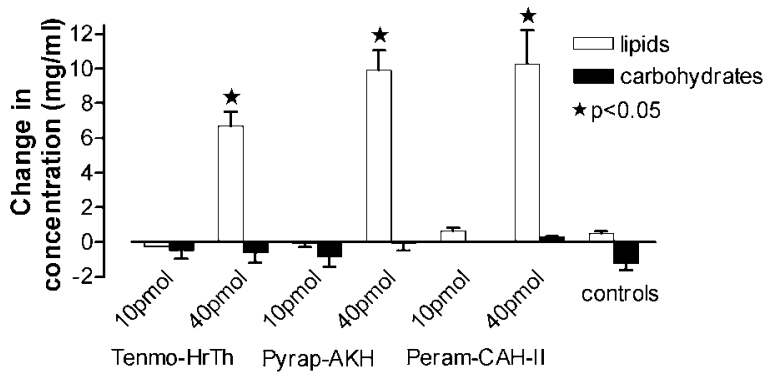

Dose of AKH peptide

Fig. 1. The effect of AKH-peptides on elevation of haemolymph lipid and carbohydrate levels $(n=8-10)$ after combinatory inter-species injections of AKHs. Controls $(n=5-10)$ were injected with saline only. Significant differences at the $5 \%$ level (experimental vs. control) are indicated by asterisks.

\section{Data presentation and statistical analysis}

The obtained results were plotted by means of the graphic program Prism (GraphPad Software, version 4.0, San Diego, CA, USA). The bar graphs represent the mean of measurements \pm SEM; the line for Fig. 4 was calculated from the two site binding hyperbola equation. Common Student's t-test was used to determine the significance of the results.

\section{RESULTS}

\section{Conspecific applications of AKHs}

In the first step of our experiments we performed the conspecific tests including four different AKHs (LocmiAKH-I, Tenmo-HrTH, Pyrap-AKH and Peram-CAH-II) and three insect species (L. migratoria, T. molitor and $P$. apterus). The results showed that Locmi-AKH-I induced hyperlipaemia and hyperglycemia in L. migratoria, Tenmo-HrTH induced hypertrehalosemia in T. molitor, and Pyrap-AKH and Peram-CAH-II induced hyperli-

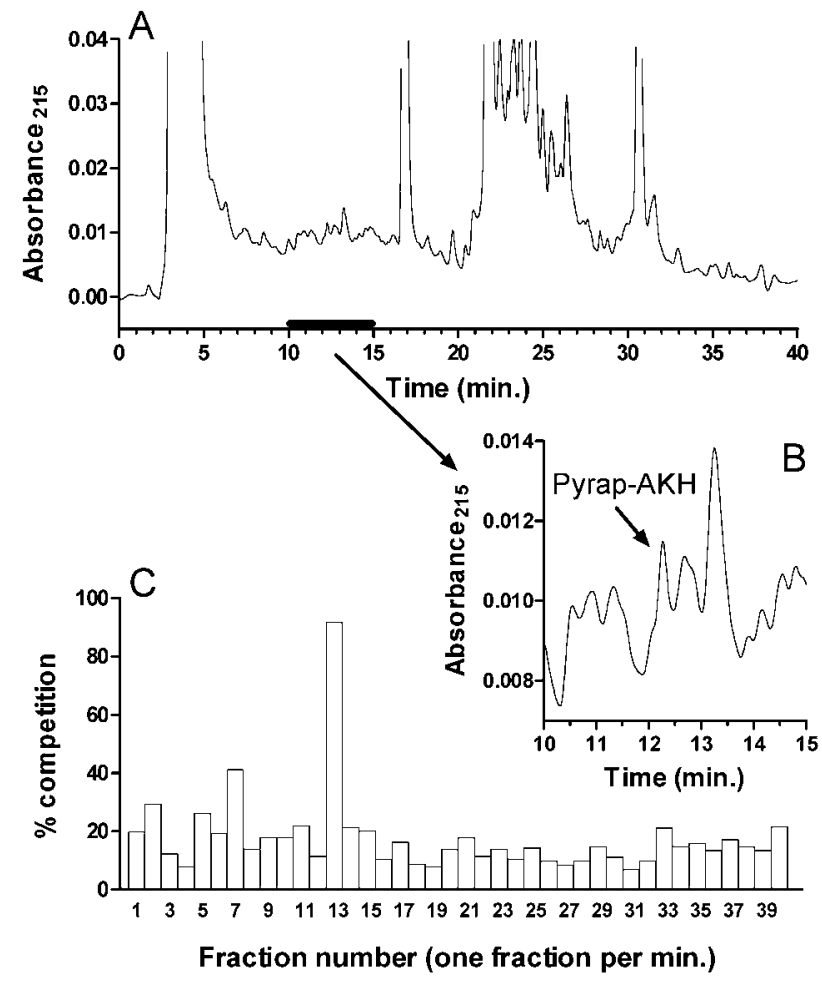

Fig. 2. The RP-HPLC elution profile of methanolic extract of 50 brain-CC-CA complexes from $D$. cingulatus (A) and the detail of the profile, where AKHs are expected to elute (B). Activity of all eluted fractions recognised by $\mathrm{Cys}^{1}$-Pyrap-AKH antibody (C): results are expressed as \% of competition of a natural AKH peptide with the BLAM-Cys ${ }^{1}$-Pyrap-AKH in competitive ELISA test (for detailed fragmentation scheme see Kodrík et al., 2000).

paemia in $P$. apterus (Table 1). Mobilization of lipid reserves in $T$. molitor was negligible, as was mobilization of carbohydrates in $P$. apterus females. Despite the fact that injection of Locmi-AKH-I activated both lipid and carbohydrate stores in L. migratoria, the hyperlipaemic effect was much more significant. The results also showed that AKHs trigger the particular mobilization pathways leading to activation of energy reserves characteristic of the test species.

\section{Inter-species applications of AKHs}

In the next series of experiments we tested the hypothesis that AKHs originating from different insects can mobilize only recipient-specific types of energy stores. We injected the same set of hormones into the same set of insect species, but in all possible heterologous hormone-species combinations. The pattern of results (Fig. 1) was very similar to the previous experiment: the treated species (recipients) mobilized the same energy source as they did after injection of their own AKHs: $P$. apterus and L. migratoria mobilized lipids and T. molitor activated carbohydrate stores.

\section{Isolation and characterization of AKH from $D$. cingulatus}

The above results led us to the conclusion that the mobilization of an energy source does not depend on the 

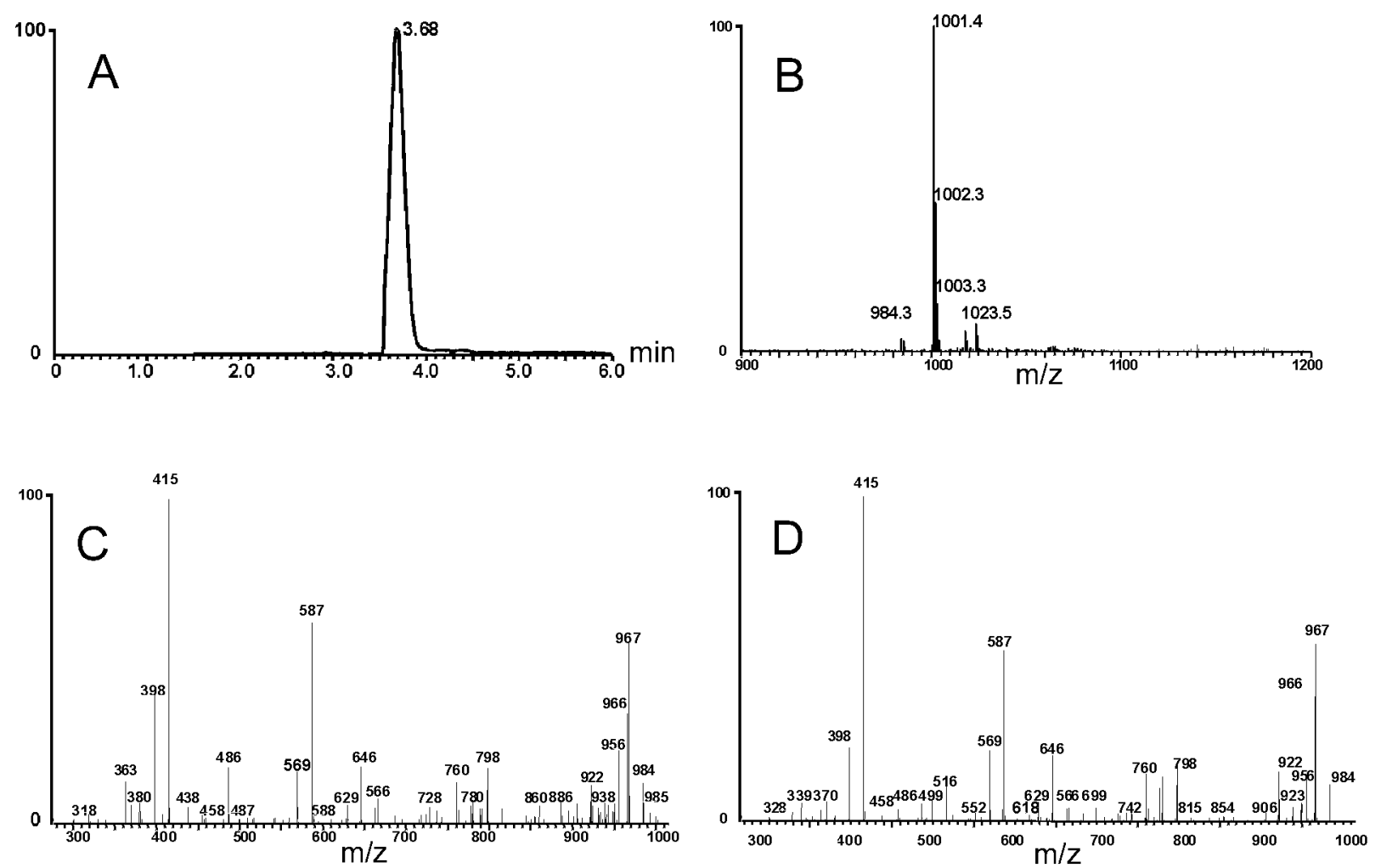

Fig. 3. Mass chromatogram of $\mathrm{MH}^{+}=1001.4$ peptide reconstructed from LC/MS analysis of both fraction No. 13 from Fig. 2 and brain-CC-CA methanolic extract of the bug $D$. cingulatus (A); averaged full scan ES mass spectrum of the peak RT $=3.68$ min from A (B); CID MS ${ }^{2}$ spectrum of the native precursor ion $\mathrm{MH}^{+}=1001.4$ from the bug $D$. cingulatus (C); CID MS ${ }^{2}$ spectrum of the precursor ion $\mathrm{MH}^{+}=1001.4$ from the reference synthetic Pyrap-AKH peptide (D).

type of AKH, but is determined by species-specific biochemical pathways. To verify this hypothesis we decided to use the bug $D$. cingulatus, a species for which the AKH(s) had not yet been characterized. RP-HPLC analysis of the methanolic brain-CC-CA extract from $D$. cingulatus generated a number of UV-absorbing peaks (Fig. 2A,B) which were tested by ELISA assay (Fig. 2C). The antibody recognised fraction No. 13, which indicated the presence of $\mathrm{AKH}$ material and moreover an $\mathrm{AKH}$ that is similar to Pyrap-AKH (see Goldsworthy et al., 2002b) against which the antibody was raised. This active frac-

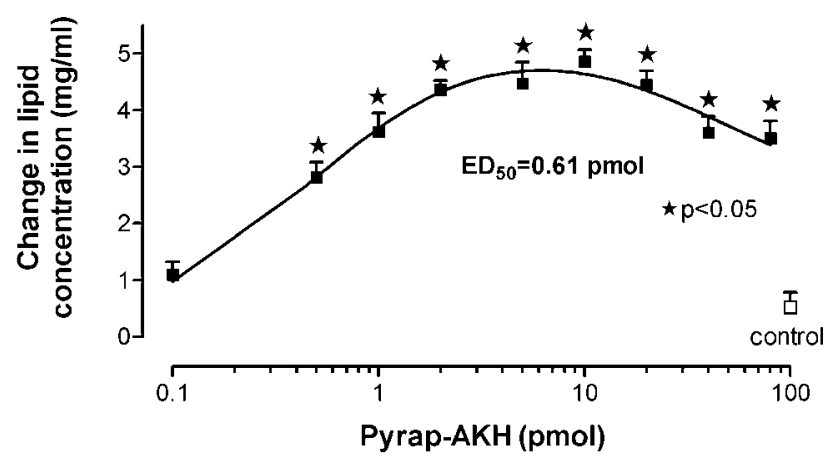

Fig. 4. The effect of increasing doses of synthetic Pyrap-AKH on the elevation of haemolymph lipid level $(n=8-10)$ in $D$. cingulatus 8-day old females. Controls $(\mathrm{n}=10)$ were injected with saline only. Significant differences at the 5\% level (experimental vs. control) are indicated by asterisks. tion, plus a parallel prepared crude methanolic brainCC-CA extract, was subjected to LC/ESI/MS ${ }^{\mathrm{N}}$ analyses (Fig. 3). Both analyses resulted in identification of an octapeptide with a mass of $1000.4 \mathrm{Da}$ and the structure pGlu-Leu-Asn-Phe-Thr-Pro-Asn-Trp- $\mathrm{NH}_{2}$. The peptide exhibited identical chromatographic and spectral properties to the previously described Pyrap-AKH isolated from the related pyrrhocorid, P. apterus (Kodrík et al., 2000). Contrary to two adipokinetic peptides isolated from the bug $P$. apterus (Kodrík et al., 2000, 2002), only the Pyrap-AKH was detected in the CNS of $D$. cingulatus. Finding of Pyrap-AKH in the latter bug is not so surprising because both $D$. cingulatus and $P$. apterus belong to the same family Pyrrhocoridae. Injection of synthetic Pyrap-AKH into D. cingulatus caused a hyperlipaemic effect - the dose response curve (Fig. 4) revealed the $\mathrm{ED}_{50}$ to be 0.61 pmol.

\section{Confirmation of the predictability hypothesis}

The last section of our experiments, aimed at confirming the validity of our hypothesis concerning the predictability of metabolic function of $\mathrm{AKH}$, included heterologous applications of four AKHs to D. cingulatus (Fig. 5). All injected AKHs mobilized lipid stores in this species, while activation of carbohydrates was either nonsignificantly less (Locmi-AKH-I), or negligible (PyrapAKH, Tenmo-HrTh, Peram-CAH-II). The results show 


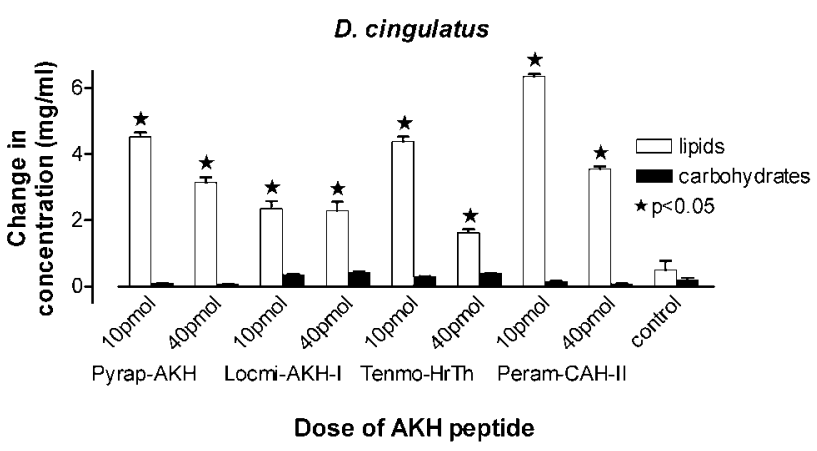

Fig. 5. The effect of all tested AKH-peptides on elevation of haemolymph lipid and carbohydrate levels $(n=8-10)$ after injection into $D$. cingulatus 8-day old females. Controls (both $\mathrm{n}$ $=10$ ) were injected with saline only. Significant differences at the $5 \%$ level (experimental vs. control) are indicated by asterisks.

that activation of lipids is the preferable metabolic pathway used in this bug.

\section{DISCUSSION}

These results and an examination of the literature revealed that AKHs of the same structure can mobilize different energy resources in different insect species. For example, while the Peram-CAH-II is known to mobilize carbohydrates in the cockroaches $P$. americana and Blatta orientalis (Witten et al., 1984; Scarborough et al., 1984), in the Colorado potato beetle Leptinotarsa decemlineata the AKH mobilizes carbohydrates and induces proline synthesis (Gäde \& Kellner, 1989), and in P. apterus it is involved in mobilization of lipids only (Kodrík et al., 2002). A similar situation can also be found with Peram-CAH-I. While in P. americana and $B$. orientalis it leads to mobilization of carbohydrates (Witten et al., 1984; Gäde \& Rinehart, 1990), in L. decemlineata (Weeda, 1981; Gäde \& Kellner, 1989) and the cerambycid beetles Ceroplesis thunbergii and Phryneta spinator (Gäde \& Auerswald, 2000) it is involved in proline synthesis and mobilization of carbohydrates. Similarly, Manse-AKH mobilizes lipids in the silkmoth, Bombyx mori (Ishibashi et al., 1992) and butterfly, Vanessa cardui (Köllisch et al., 2000), carbohydrate in the noctuid moth, Heliothis zea (Jaffe et al., 1986), and both lipids and carbohydrates in the tobacco hornworm moth, Manduca sexta (Ziegler et al., 1985). SchgrAKH-II functions as a hyperlipaemic agent, for example in the pyrgomorphid grasshopper, Phymateus morbillosus (Gäde et al., 1996), but mobilizes carbohydrates in the armoured ground cricket, Acanthoproctus cervinus (Gäde, 1992). The question whether the usage of different energy substrates in different insect species with the same structure of native AKH is coupled with the evolution of different species-specific types of AKH receptors responsible for various signal transduction events remains to be answered. It is noteworthy to mention that the use of carbohydrates and proline occurs commonly in some beetles and flies, and that dual usage of proline and carbohydrate metabolism might be mediated by different receptors, or by receptor subtypes (Auerswald \& Gäde, 1999). Dual use of carbohydrates and lipids seems to be more conserved because both locust (Beenakkers et al., 1981) and moths (Ziegler \& Schulz, 1986a, b) use carbohydrates and lipids to power flight activity. It remains to be discovered, if there are also insects using both proline and lipid metabolic pathways simultaneously. Nevertheless, the use of proline can be interpreted as an alternative - probably more specialized - variant of lipid consumption (Gäde \& Auerswald, 2003). Provided that the latter view is correct, then during the evolution of some insect species, the original dual usage of carbohydrate and lipids was substituted by dual usage of carbohydrates and proline.

All the data mentioned above, additional to those from the present study, demonstrate that metabolic responses of AKHs are characteristic of the recipient species rather than of the structure of the AKH tested and that the type of AKH-mobilized energy substrates in a tested insect can be predicted irrespective of the form of active AKH being applied. Therefore, the metabolic function in tested species cannot be predicted from the metabolic responses obtained in acceptor insect species to which crude CC extracts from a test species were applied, i.e. from the metabolic activities found in Locusta and Periplaneta heterologous bioassays. Thus, it seems to be misleading to speak about hyperlipaemic, hypertrehalosaemic and hyperprolinaemic factor(s) in crude CC extracts of a test insect on the basis of the results obtained from the Locusta and Periplaneta heterologous bioassays.

Recently we have identified two native AKHs (Pyrap-AKH and Peram-CAH-II) in the firebug, $P$. apterus (Kodrík et al., 2000, 2002), in which they mobilized lipid stores but had no effect on carbohydrates. Provided that the conclusions of three different AKHs receptors in L. migratoria (Vroemen et al., 1998) and the existence of two or more receptors in all insects (Staubli et al., 2002) are correct, then at least two main types of AKH receptors involved in the actions of Pyrap-AKH and Peram-CAH-II have yet to be identified in P. apterus. There is a good circumstantial evidence for the existence of different populations of $\mathrm{AKH}$-receptors in the insect fat body (Lee \& Goldsworthy, 1998).

In summary, the present study revealed the structure and metabolic function of the native AKH in D. cingulatus and showed that the metabolic function of AKH can be predicted without knowledge of its structure but only on the basis of results obtained from the heterologous bioassays. We propose that such an approach can serve as a quick monitoring system for screening the energy substrates mobilized by native insect AKHs until their structures are identified.

ACKNOWLEDGEMENTS. This study was supported by the Institute of Entomology project No. Z5007907 (Academy of Sciences of the Czech Republic), and by grants No. A6007202 from the Grant Agency of the Czech Academy of Sciences (DK) and No. 206/03/0016 from the Grant Agency of the Czech Republic (RS). The authors thank H. Radová and D. Rienesslová for their technical assistance. 


\section{REFERENCES}

Auerswald L. \& GÄDE G. 1999: Effects of metabolic neuropeptides from insect corpora cardiaca on proline metabolism of the African fruit beetle, Pachnoda sinuata. J. Insect Physiol. 45: 535-543.

BECKER M. \& WegENER G. 1998: Hypetrehalosaemic neuropeptides decrease levels of the glycolytic signal fructose 2,6-bisphosphate in cockroach fat body. J. Exp. Biol. 201: 1939-1946.

Beenakkers A.M.T., van der Horst D.J. \& van MarrewiJK W.J.A. 1981: Metabolism during locust flight. Comp. Biochem. Physiol.(B) 69: 315-321.

BeEnakKers A.M.T., van der Horst D.J. \& van MarrewiJk W.J.A. 1984: Insect flight muscle metabolism. Insect Biochem. 14: 243-260.

Carlisle J. \& Loughton B.G. 1979: Adipokinetic hormone inhibits protein synthesis in Locusta. J. Insect Physiol. 282: 420-421.

Cusinato O., Wheeler C.H. \& Goldsworthy G.J. 1991: The identity and physiological actions of an adipokinetic hormone in Acheta domesticus. J. Insect Physiol. 37: 461-469.

Ebberink R.H.M., Smit A.B. \& VAn Minnen J. 1989: The insulin family, evolution of structure and function in vertebrates and invertebrates. Biol. Bull. 177: 176-182.

GÄDE G. 1992: Isolation and structure elucidation of neuropeptides of the AKH/RPCH family in long-horned grasshoppers (Ensifera). Biol. Chem. Hoppe-Seyler 373: 1169-1178.

GäDE G. \& AuERSwALD L. 1998: Insect neuropeptides regulating substrate mobilisation. S. Afr. J. Zool. 33: 65-77.

Gäde G. \& AuERswald L. 2000: Flight substrates and their regulation by a member of the AKH/RPCH family of neuropeptides in Cerambycidae. J. Insect Physiol. 46: 1575-1564.

GädE G. \& AuERSWALd L. 2003: Mode of action of neuropeptides from the adipokinetic hormone family. Gen. Comp. Endocrinol. 132: 10-20.

GäDE G. \& Kellner R. 1989: The metabolic neuropeptides of the corpus cardiacum from the potato beetle and the American cockroach are identical. Peptides 10: 1287-1289.

GÄDE G. \& RineharT K.L. 1990: Primary structures of hypertrehalosaemic neuropeptides isolated from the corpora cardiaca of the cockroaches Leucophaea maderae, Gromphadorhina portentosa, Blattella germanica and Blatta orientalis and of the stick insect Extatosoma tiaratum assigned by tandem fast atom bombardment mass spectrometry. Biol. Chem. HoppeSeyler 371: 345-354.

Gäde G., Kellner R. \& Rinehart K.L. 1996: Pyrgomorphid grasshoppers of the genus Phymateus contain speciesspecific decapeptides of the AKH/RPCH family regulating lipid-mobilization during flight. Physiol. Entomol. 21: 193-202.

Gäde G., Hoffmann K.H. \& Spring J.H. 1997: Hormonal regulation in insects: facts, gaps, and future directions. Physiol. Rev. 77: $963-1032$.

Gokuldas M., Hunt A.P. \& Candy D.J. 1988: The inhibition of lipid synthesis in vitro in the locust, Schistocerca gregaria, by factors from the corpora cardiaca. Physiol. Entomol. 13: $43-48$.

Goldsworthy G.J. 1983: The endocrine control of flight metabolism in locusts. Adv. Insect Physiol. 17:149-204.

Goldsworthy G., Opoku-Ware K. \& Mullen L. 2002a: Adipokinetic hormone enhances laminarin and bacterial lipopolysaccharide-induced activation of the prophenoloxidase cascade in the African migratory locust, Locusta migratoria. J. Insect Physiol. 48: 601-608.
Goldsworthy G.J., Kodrík D., Comley R. \& Lightfoot M. 2002b: A quantitative study of the adipokinetic hormone of the firebug, Pyrrhocoris apterus. J. Insect Physiol. 48: 1103-1109.

Goldsworthy G., Mullen L., Opoku-Ware K. \& Chandrakant S. 2003: Interactions between the endocrine and immune systems in locusts. Physiol. Entomol. 28: 54-61.

Ishibashi J., Kataoka H., Nagasawa H., Isogai A. \& Suzuki A. 1992: Isolation and identification of adipokinetic hormone of the silkworm, Bombyx mori. Biosci. Biotech. Biochem. 56: 66-70.

Jaffe H., Raina A.K., Riley C.T., Fraser B.A., Holman G.M., WAGNeR R.M., Ridgway R.L. \& HAYes D.K. 1986: Isolation and primary structures of a peptide from the corpora cardiaca of Heliothis zea with adipokinetic activity. Biochem. Biophys. Res. Commun. 135: 622-628.

KodRík D. \& Goldsworthy G.J. 1995: Inhibition of RNA synthesis by adipokinetic hormones and brain factor(s) in adult fat body of Locusta migratoria. J. Insect Physiol. 41: 127-133.

Kodrík D., ŠImeK P., LepŠA L. \& Socha R. 2002: Identification of the cockroach neuropeptide Pea-CAH-II as a second adipokinetic hormone in the firebug Pyrrhocoris apterus. Peptides 23: 583-585.

Kodrík D., Socha R., Šimek P., Zemek R. \& Goldsworthy G.J. 2000: A new member of the AKH/RPCH family that stimulates locomotory activity in the firebug, Pyrrhocoris apterus (Heteroptera). Insect Biochem. Mol. Biol. 30: 489-498.

Köllisch V.G., Lorenz M.W., Kellner R., Verhaert P.D. \& HofFMAnN K.H. 2000: Structure elucidation and biological activity of an unusual adipokinetic hormone from corpora cardiaca of the butterfly, Vanessa cardui. Eur. J. Biochem. 267: $5502-5508$.

LAFONT R. 2000: The endocrinology of invertebrates. Ecotoxicology 9: 41-57.

Lee M. \& Goldsworthy G.J. 1998: New perspectives on the structures, assays and actions of locust adipokinetic hormones. In Coast G.M. \& Webster S. (eds): Recent Advances in Arthropod Endocrinology: SEB Seminar Series. Cambridge University Press, Cambridge, UK, pp. 149-171.

LORENZ M.W. 2001: Synthesis of lipids in the fat body of Gryllus bimaculatus: age-dependency and regulation by adipokinetic hormone. Arch. Insect Biochem. Physiol. 47: 198-214.

Milde J.J., Ziegler R. \& Wallstein M. 1995: Adipokinetic hormone stimulates neurones in the central nervous system. $J$. Exp. Biol. 198: 1307-1311.

Montgomery R. 1957: Determination of glycogen. Arch. Biochem. Biophys. 67: 378-386.

Satake H., Takuwa K., Minakata H. \& Matsushima O. 1999: Evidence for conservation of the vasopressin/oxytocin superfamily in annelids. J. Biol. Chem. 274: 5605-5611.

Scarborough R.M., Jamieson G.C., Kalish F., Kramer S.J., McEnroe G.A., Miller C.A. \& Schooley D.A. 1984: Isolation and primary structure of two peptides with cardioacceleratory and hyperglycemic activity from the corpora cardiaca of Periplaneta americana. Proc. Natl. Acad. Sci. USA 81: 5575-5579.

Socha R. \& KodRík D. 1999: Differences in adipokinetic response of Pyrrhocoris apterus (Heteroptera) in relation to wing dimorphism and diapause. Physiol. Entomol. 24: 278-284.

SоснA R. \& Šula J. 1996: Differences in haemolymph proteins in relation to diapause and wing dimorphism in Pyrrhocoris apterus (Heteroptera: Pyrrhocoridae). J. Comp. Physiol. (B) 166: $382-376$. 
Socha R., KodRíK D. \& ZEMEK R. 1999: Adipokinetic hormone stimulates insect locomotor activity. Naturwissenschaften $\mathbf{8 8}$ : $85-86$.

Staubli F., Jorgensen T.J.D., Cazzamali G., Williamson M., Lenz C., Sondergaard L., Roepstorff P. \& Grimmelikhuijzem C.J.P. 2002: Molecular identification of the insect adipokinetic hormone receptors. Proc. Natl. Acad. Sci. USA 99: 3446-3451.

VRoemen S.F., Van de Horst D.J. \& Van MarrewiJK W.J.A. 1998: At the cutting edge. New insights into adipokinetic hormone signalling. Mol. Cell. Endocr. 141: 7-12.

WeEda E., DeKort C.A.D. \& Beenakkers A.M.T. 1979: Fuels for energy metabolism in the Colorado potato beetle, Leptinotarsa decemlineata Say. J. Insect Physiol. 25: 951-955.

WEEDA E. 1981: Hormonal regulation of proline synthesis and glucose release in the fat body of the Colorado potato beetle, Leptinotarsa decemlineata. J. Insect Physiol. 27: 411-417.

Witten J.L., Schaffer M.H., O'shea M., Cook J.C., Hemling M.E. \& RineharT K.L., JR. 1984: Structure of two cockroach neuropeptides assigned by fast atom bombardment mass spectrometry. Biochem. Biophys. Res. Commun. 124: 350-358.
ZiEGLER R. 1997: Lipid synthesis by ovaries and fat body of Aedes aegypti (Diptera: Culicidae). Eur. J. Entomol. 94: 385-391.

ZIEGLER R. \& SChULZ M. 1986a: Regulation of carbohydrate metabolism during flight in Manduca sexta. J. Insect Physiol. 32: 997-1001

Ziegler R. \& SChUlz M. 1986b: Regulation of lipid metabolism during flight in Manduca sexta. J. Insect Physiol. 32: 903-908.

Ziegler R., Eckart K., Schwarz H. \& Keller R. 1985: Aminoacid sequence of Manduca sexta adipokinetic hormone elucidated by combined fast atom bombardment (FAB). Biochem. Biophys. Res. Commun. 133: 337-342.

ZöLLNER N. \& KIRSCH K. 1962: Über die quantitative Bestimmung von Lipoide (Mikromethode) mittels der vielen natürlichen Lipoiden (allen bekannten Plasmalipoiden) gemeinsamen Sulfo- phospho-vanillin-Reaktion. Zeitschr. f. Gesam. Exp. Med. 135: 545-561.

Received November 4, 2003; revised December 12, 2003; accepted January 22, 2004 